\title{
THE CURSE OF DIMENSIONALITY FOR NUMERICAL INTEGRATION OF SMOOTH FUNCTIONS
}

\author{
A. HINRICHS, E. NOVAK, M. ULLRICH, AND H. WOŹNIAKOWSKI
}

\begin{abstract}
We prove the curse of dimensionality for multivariate integration of $C^{r}$ functions: The number of needed function values to achieve an error $\epsilon$ is larger than $c_{r}(1+\gamma)^{d}$ for $\epsilon \leq \epsilon_{0}$, where $c_{r}, \gamma>0$. The proofs are based on volume estimates for $r=1$ together with smoothing by convolution. This allows us to obtain smooth fooling functions for $r>1$.
\end{abstract}

\section{INTRODUCTION}

We study multivariate integration for different classes $F_{d}$ of smooth functions $f: \mathbb{R}^{d} \rightarrow \mathbb{R}$. Our emphasis is on large values of $d \in \mathbb{N}$. We want to approximate

$$
S_{d}(f)=\int_{D_{d}} f(x) \mathrm{d} x \text { for } f \in F_{d}
$$

up to some error $\varepsilon>0$, where $D_{d} \subset \mathbb{R}^{d}$ has Lebesgue measure 1 . The results in this paper hold for arbitrary sets $D_{d}$, the standard example of course is $D_{d}=[0,1]^{d}$.

We consider (deterministic) algorithms that use only function values, and classes $F_{d}$ of functions bounded in absolute value by 1 and containing all constant functions $f(x) \equiv c$ with $|c| \leq 1$. An algorithm that uses no function value at all must be a constant, $A_{0}(f) \equiv b$, and its error is at least

$$
\max _{f \in F_{d}}\left|S_{d}(f)\right|=1 \text {. }
$$

We call this the initial error of the problem, it does not depend on $d$. Hence multivariate integration is well scaled and that is why we consider $\varepsilon<1$.

Let $n\left(\varepsilon, F_{d}\right)$ denote the minimal number of function values needed for this task in the worst case setting 1 . By the curse of dimensionality we mean that $n\left(\varepsilon, F_{d}\right)$ is exponentially large in $d$. That is, there are positive numbers $c, \varepsilon_{0}$ and $\gamma$ such that

$$
n\left(\varepsilon, F_{d}\right) \geq c(1+\gamma)^{d} \quad \text { for all } \varepsilon \leq \varepsilon_{0} \quad \text { and infinitely many } \quad d \in \mathbb{N} .
$$

For many natural classes $F_{d}$ the bound in (2) will hold for all $d \in \mathbb{N}$. This applies in particular to the classes considered in this paper.

Received by the editor November 5, 2012 and, in revised form, April 16, 2013.

2010 Mathematics Subject Classification. Primary 65D30, 65Y20, 41A63, 41A55.

Key words and phrases. Curse of dimensionality, numerical integration, high dimensional numerical problems.

The first author was partially supported by the DFG-Priority Program 1324.

The third author was supported by DFG GRK 1523 and ERC Advanced Grant PTRELSS.

The fourth author was partially supported by the National Science Foundation.

${ }^{1}$ We add that $n\left(\varepsilon, F_{d}\right)$ is the information complexity of multivariate integration over $F_{d}$ and is proportional to the (total) complexity as long as $F_{d}$ is convex and symmetric. The last two assumptions are needed to guarantee that a linear algorithm is optimal and its implementation cost is linear in $n\left(\varepsilon, F_{d}\right)$. 
There are many classes $F_{d}$ for which the curse of dimensionality has been proved; see [5, 7. for such examples. However, it has not been known if the curse of dimensionality occurs for probably the most natural class which is the unit ball of $r$ times continuously differentiable functions,

$$
\mathcal{C}_{d}^{r}=\left\{f \in C^{r}\left(\mathbb{R}^{d}\right) \mid\left\|D^{\beta} f\right\| \leq 1 \quad \text { for all }|\beta| \leq r\right\},
$$

where $\beta=\left(\beta_{1}, \beta_{2}, \ldots, \beta_{d}\right)$, with nonnegative integers $\beta_{j},|\beta|=\sum_{j=1}^{d} \beta_{j}$, and $D^{\beta}$ denotes the operator of $\beta_{j}$ times differentiation with respect to the $j$ th variable for $j=1,2, \ldots, d$. By $\|\cdot\|$ we mean the sup norm, $\left\|D^{\beta} f\right\|=\sup _{x \in \mathbb{R}^{d}}\left|\left(D^{\beta} f\right)(x)\right|$.

For $r=0$, we obviously have $n\left(\varepsilon, \mathcal{C}_{d}^{0}\right)=\infty$ for all $\varepsilon<1$ and all $d \in \mathbb{N}$. Therefore, from now on we always assume that $r \geq 1$. For $r=1$, the curse of dimensionality for $\mathcal{C}_{d}^{1}$ follows from the results of Sukharev [8]. Whether the curse holds for $r \geq 2$ has been an open problem for many years.

The class $\mathcal{C}_{d}^{r}$ for $D_{d}=[0,1]^{d}$ (and functions and norms restricted to $D_{d}$ ) was already studied in 1959 by Bakhvalov [2]; see also 4. He proved that there are two positive numbers $a_{d, r}$ and $A_{d, r}$ such that

$$
a_{d, r} \varepsilon^{-d / r} \leq n\left(\varepsilon, \mathcal{C}_{d}^{r}\right) \leq A_{d, r} \varepsilon^{-d / r} \quad \text { for all } d \in \mathbb{N} \text { and } \varepsilon \in(0,1) .
$$

This means that for a fixed $d$ and for $\varepsilon$ tending to zero, we know that $n\left(\varepsilon, \mathcal{C}_{d}^{r}\right)$ is of order $\varepsilon^{-d / r}$ and the exponent of $\varepsilon^{-1}$ grows linearly in $d$. Unfortunately, Bakhvalov's result does not allow us to conclude whether the curse of dimensionality holds for the class $\mathcal{C}_{d}^{r}$. In fact, if we reverse the roles of $d$ and $\varepsilon$, and consider a fixed $\varepsilon$ and $d$ tending to infinity, the bound (3) on $n\left(\varepsilon, \mathcal{C}_{d}^{r}\right)$ is useless. We prove the following result and hereby solve Open Problem 1 from [5]:

Main Theorem. The curse of dimensionality holds for the classes $\mathcal{C}_{d}^{r}$ with the super-exponential lower bound

$$
n\left(\varepsilon, \mathcal{C}_{d}^{r}\right) \geq c_{r}(1-\varepsilon) d^{d /(2 r+3)} \quad \text { for all } d \in \mathbb{N} \text { and } \varepsilon \in(0,1),
$$

where $c_{r} \in(0,1]$ depends only on $r$.

We also prove that the curse of dimensionality holds for even smaller classes of functions $F_{d}$ for which the norms of arbitrary directional derivatives are bounded proportionally to $1 / \sqrt{d}$.

We now discuss how we obtain lower bounds on $n\left(\varepsilon, F_{d}\right)$ for numerical integration defined on convex and symmetric classes $F_{d}$. The standard proof technique is to find a fooling function $f \in F_{d}$ that vanishes at the points $\mathcal{P}=\left\{x_{1}, x_{2}, \ldots, x_{n}\right\}$ at which we sample functions from $F_{d}$, and the integral of $f$ is as large as possible. All algorithms that use function values at $x_{j}$ 's must give the same approximation of the integral for $f$ and $-f$. Thus, each such algorithm makes an error of at least $\left|S_{d}(f)-S_{d}(-f)\right| / 2=\left|S_{d}(f)\right|$ for one of the functions. That is why the integral of $f$ is a lower bound on the worst case error of all algorithms using function values at $x_{j}$ 's. If, for all choices of $x_{1}, x_{2}, \ldots, x_{n}$, there are functions $f \in F_{d}$ vanishing at $x_{j}$ 's with integrals larger than $\varepsilon$, then $n\left(\varepsilon, F_{d}\right) \geq n$.

We start with the fooling function

$$
f_{0}(x)=\min \left\{1, \frac{1}{\delta \sqrt{d}} \operatorname{dist}\left(x, \mathcal{P}_{\delta}\right)\right\} \quad \text { for all } \quad x \in \mathbb{R}^{d}
$$


where

$$
\mathcal{P}_{\delta}=\bigcup_{i=1}^{n} B_{\delta}^{d}\left(x_{i}\right)
$$

and $B_{\delta}^{d}\left(x_{i}\right)$ is the ball with center $x_{i}$ and radius $\delta \sqrt{d}$. The function $f_{0}$ is Lipschitz. By a suitable smoothing via convolution we construct a fooling function $f_{r} \in \mathcal{C}_{d}^{r}$ with $\left.f_{r}\right|_{\mathcal{P}}=0$.

\section{Preliminaries}

In this section, we precisely define our problem. Let $F_{d}$ be a class of continuous functions $f: \mathbb{R}^{d} \rightarrow \mathbb{R}$ such that $S_{d}(f)$ (see (1)) exists for every $f \in F_{d}$. We approximate the integral $S_{d}(f), f \in F_{d}$, by algorithms

$$
A_{n, d}(f)=\phi_{n, d}\left(f\left(x_{1}\right), f\left(x_{2}\right), \ldots, f\left(x_{n}\right)\right),
$$

where $x_{j} \in \mathbb{R}^{d}$ can be chosen adaptively and $\phi_{n, d}: \mathbb{R}^{n} \rightarrow \mathbb{R}$ is an arbitrary mapping. Adaption means that the selection of $x_{j}$ may depend on the already computed values $f\left(x_{1}\right), f\left(x_{2}\right), \ldots, f\left(x_{j-1}\right)$. The (worst case) error of the algorithm $A_{n, d}$ is defined as

$$
e\left(A_{n, d}\right)=\sup _{f \in F_{d}}\left|S_{d}(f)-A_{n, d}(f)\right| .
$$

The minimal number of function values to guarantee that the error is at most $\varepsilon$ is defined as

$$
n\left(\varepsilon, F_{d}\right)=\min \left\{n \in \mathbb{N} \mid \exists A_{n, d} \text { such that } e\left(A_{n, d}\right) \leq \varepsilon\right\} .
$$

Hence we minimize $n$ over all choices of adaptive sample points $x_{j}$ and mappings $\phi_{n, d}$. It is well known that, as long as the class $F_{d}$ is convex and symmetric, we may restrict the minimization of $n$ by considering only nonadaptive choices of $x_{j}$ and linear mappings $\phi_{n, d}$. Furthermore,

$$
n\left(\varepsilon, F_{d}\right)=\min \left\{n \in \mathbb{N}\left|\inf _{\mathcal{P} \subset \mathbb{R}^{d}, \# \mathcal{P}=n} \sup _{f \in F_{d},\left.f\right|_{\mathcal{P}}=0}\right| S_{d}(f) \mid \leq \varepsilon\right\} ;
$$

see [4, Prop. 1.2.6] or [9, Theorem 5.5.1]. In this paper, we always consider convex and symmetric $F_{d}$ so that we can use the last formula for $n\left(\varepsilon, F_{d}\right)$. For more details see, e.g., Chapter 4 in [5].

As already mentioned, our lower bounds are based on a volume estimate of a neighborhood of certain sets in $\mathbb{R}^{d}$; see also $\left[3\right.$. In the following, we denote by $A_{\delta}$ the $(\delta \sqrt{d})$-neighborhood of $A \subset \mathbb{R}^{d}$, which is defined by

$$
A_{\delta}=\left\{x \in \mathbb{R}^{d} \mid \operatorname{dist}(x, A) \leq \delta \sqrt{d}\right\},
$$

where $\operatorname{dist}(x, A)=\inf _{a \in A}\|x-a\|_{2}$ denotes the Euclidean distance of $x$ from $A$.

Furthermore, we denote by $B_{\delta}^{d}(x)$ the $d$-dimensional ball with center $x \in \mathbb{R}^{d}$ and radius $\delta \sqrt{d}$, i.e.,

$$
B_{\delta}^{d}(x)=\left\{y \in \mathbb{R}^{d} \mid\|x-y\|_{2} \leq \delta \sqrt{d}\right\} .
$$

We will need some standard volume estimates for Euclidean balls. Recall that the volume of a Euclidean ball of radius 1 is given by

$$
V_{d}=\frac{\pi^{d / 2}}{\Gamma(1+d / 2)}
$$


From Stirling's formula for the $\Gamma$ function, we have

$$
\Gamma(x+1)=\sqrt{2 \pi x} x^{x} \mathrm{e}^{-x+\frac{\theta_{x}}{12 x}} \quad \text { for all } \quad x>0,
$$

where $\theta_{x} \in(0,1)$; see [1, p. 257]. This leads to the estimate

$$
\Gamma(x+1)>\sqrt{2 \pi x}\left(\frac{x}{\mathrm{e}}\right)^{x} \quad \text { for all } \quad x>0 .
$$

Combining this estimate with the volume formula for the ball, we obtain for all $d \in \mathbb{N}$,

$$
\lambda_{d}\left(B_{\delta}^{d}(x)\right)=(\delta \sqrt{d})^{d} V_{d}<(\delta \sqrt{d})^{d} \frac{\left(\frac{2 \pi \mathrm{e}}{d}\right)^{d / 2}}{\sqrt{\pi d}}=\frac{(\delta \sqrt{2 \pi \mathrm{e}})^{d}}{\sqrt{\pi d}}<(\delta \sqrt{2 \pi \mathrm{e}})^{d},
$$

where $\lambda_{d}$ is the Lebesgue measure. The volume formula for the Euclidean unit ball also shows the recurrence relation

$$
\frac{V_{d-1}}{V_{d}}=\frac{d}{d-1} \frac{V_{d-3}}{V_{d-2}} \quad \text { for all } \quad d \geq 4
$$

This easily implies

$$
\frac{2}{\sqrt{d}} \frac{V_{d-1}}{V_{d}}<\frac{2}{\sqrt{d-2}} \frac{V_{d-3}}{V_{d-2}} \text { for all } \quad d \geq 4 .
$$

The last inequality can be used in an inductive argument leading to

$$
\frac{2}{\sqrt{d}} \frac{V_{d-1}}{V_{d}} \leq 1 \text { for all } d \geq 2
$$

This will be needed later.

\section{Convolution}

In this section, we fix $k \in \mathbb{N}$ and study the convolution

$$
f_{k}:=f * g_{1} * \ldots * g_{k}
$$

of a function $f$ defined on $\mathbb{R}^{d}$ with (normalized) indicator functions $g_{j}$. We are interested in properties of $f_{k}$ in terms of the properties of the initial function $f$. Recall that the convolution of two functions $f$ and $g$ on $\mathbb{R}^{d}$ is defined by

$$
(f * g)(x)=\int_{\mathbb{R}^{d}} f(x-t) g(t) \mathrm{d} t \quad \text { for all } \quad x \in \mathbb{R}^{d} .
$$

Fix a number $\delta>0$ and a sequence $\left(\alpha_{j}\right)_{j=1}^{k}$ with $\alpha_{j}>0$ such that

$$
\sum_{j=1}^{k} \alpha_{j} \leq 1
$$

For example, we may take $\alpha_{j}=1 / k$ for $j=1,2, \ldots, k$. For $j=1, \ldots, k$, we define the ball

$$
B_{j}=\left\{x \in \mathbb{R}^{d} \mid\|x\|_{2} \leq \alpha_{j} \delta \sqrt{d}\right\}
$$

and the function $g_{j}: \mathbb{R}^{d} \rightarrow \mathbb{R}$ by

$$
g_{j}(x)=\frac{\mathbb{1}_{B_{j}}(x)}{\lambda_{d}\left(B_{j}\right)}=\frac{1}{\lambda_{d}\left(B_{j}\right)} \begin{cases}1 & \text { if } x \in B_{j} \\ 0 & \text { otherwise }\end{cases}
$$


Thus, the convolution of a function $f$ with $g_{j}$ can be written as

$$
\left(f * g_{j}\right)(x)=\frac{1}{\lambda_{d}\left(B_{j}\right)} \int_{B_{j}} f(x+t) \mathrm{d} t \quad \text { for all } \quad x \in \mathbb{R}^{d} .
$$

We will frequently use the following probabilistic interpretation. Let $Y_{j}$ be a random variable that is uniformly distributed on $B_{j}$. Then the convolution of $f$ with $g_{j}$ can be written as the expected value

$$
\left(f * g_{j}\right)(x)=\mathbb{E}\left[f\left(x+Y_{j}\right)\right] .
$$

The next theorem is the basis for the induction steps of the proofs of our main results. For $f: \mathbb{R}^{d} \rightarrow \mathbb{R}$, we use the Lipschitz constant

$$
\operatorname{Lip}(f)=\sup _{x \neq y} \frac{|f(x)-f(y)|}{\|x-y\|_{2}} .
$$

Define

$$
\begin{array}{r}
C^{r}=\left\{f: \mathbb{R}^{d} \rightarrow \mathbb{R} \mid D^{\theta_{\ell}} \ldots D^{\theta_{1}} f \text { is continuous for all } \ell \leq r\right. \\
\text { and all } \left.\theta_{1}, \ldots, \theta_{r} \in \mathbb{S}^{d-1}\right\},
\end{array}
$$

where $\mathbb{S}^{d-1}$ is the unit sphere in $\mathbb{R}^{d}$ and $D^{\theta_{1}} f(x)=\lim _{h \rightarrow 0} \frac{1}{h}\left(f\left(x+h \theta_{1}\right)-f(x)\right)$ is the derivative in the direction of $\theta_{1}$.

Theorem 1. For $k \in \mathbb{N}$ and $f \in C^{r}$, define

$$
f_{k}=f * g_{1} * \ldots * g_{k} \text { with } g_{j} \text { from (8). }
$$

For $d \geq 2$, let $\Omega \subset \mathbb{R}^{d}$ and let $\Omega_{\delta}$ be its neighborhood defined as in (5). Then:

(i) if $f(x)=0$ for all $x \in \Omega_{\delta}$, then $f_{k}(x)=0$ for all $x \in \Omega$,

(ii) $\operatorname{Lip}\left(f_{k}\right) \leq \operatorname{Lip}(f)$,

(iii) if $\int_{D_{d}} f(x+t) \mathrm{d} x \geq \varepsilon$ for all $t \in \mathbb{R}^{d}$ with $\|t\|_{2} \leq \delta \sqrt{d}$, then $\int_{D_{d}} f_{k}(x) \mathrm{d} x \geq \varepsilon$,

(iv) for all $\ell \leq r$ and all $\theta_{1}, \theta_{2}, \ldots, \theta_{\ell} \in \mathbb{S}^{d-1}$,

$$
\operatorname{Lip}\left(D^{\theta_{\ell}} D^{\theta_{\ell-1}} \ldots D^{\theta_{1}} f_{k}\right) \leq \operatorname{Lip}\left(D^{\theta_{\ell}} D^{\theta_{\ell-1}} \ldots D^{\theta_{1}} f\right),
$$

(v) $f_{k} \in C^{r+k}$, and for all $\ell \leq r$, all $j=1, \ldots, k$ and all $\theta_{1}, \theta_{2}, \ldots, \theta_{\ell+j} \in \mathbb{S}^{d-1}$,

$$
\operatorname{Lip}\left(D^{\theta_{\ell+j}} D^{\theta_{\ell+j-1}} \ldots D^{\theta_{1}} f_{k}\right) \leq\left(\prod_{i=1}^{j} \frac{1}{\delta \alpha_{i}}\right) \operatorname{Lip}\left(D^{\theta_{\ell}} D^{\theta_{\ell-1}} \ldots D^{\theta_{1}} f\right) .
$$

The parts $(i)-(i v)$ of this theorem show that some properties of the initial function $f$ are preserved by convolutions. Part $(v)$ states that we gain one "degree of smoothness" with every convolution, losing only a multiplicative constant for its Lipschitz constant.

Proof. First note that we can write $f_{k}$ as

$$
f_{k}(x)=\mathbb{E}[f(x+Y)], \quad \text { for all } \quad x \in \mathbb{R}^{d},
$$

where $Y$ is a random variable with probability density function $g_{1} * \ldots * g_{k}$. By construction of $g_{j}$ 's which are the indicator functions of the balls whose sum of the radii is at most $\delta \sqrt{d}$, we have

$$
\left\{t \in \mathbb{R}^{d} \mid g_{1} * \ldots * g_{k}(t)>0\right\} \subset\left\{t \in \mathbb{R}^{d} \mid\|t\|_{2} \leq \delta \sqrt{d}\right\},
$$

which implies that $x+Y \in \Omega_{\delta}$ almost surely for every $x \in \Omega$. Thus, $f(x)=0$ for all $x \in \Omega_{\delta}$ implies that $f_{k}(x)=0$ for all $x \in \Omega$, which is property $(i)$. 
Property $(i i)$ is proven by

$$
\begin{aligned}
\left|f_{k}(x)-f_{k}(y)\right| & =|\mathbb{E}[f(x+Y)-f(y+Y)]| \leq \mathbb{E}[|f(x+Y)-f(y+Y)|] \\
& \leq \operatorname{Lip}(f) \mathbb{E}\left[\|(x+Y)-(y+Y)\|_{2}\right]=\operatorname{Lip}(f)\|x-y\|_{2} .
\end{aligned}
$$

To prove (iii), we use Fubini's theorem and we obtain

$$
\int_{D_{d}} f_{k}(x) \mathrm{d} x=\int_{D_{d}} \mathbb{E}[f(x+Y)] \mathrm{d} x=\mathbb{E}\left[\int_{D_{d}} f(x+Y) \mathrm{d} x\right] \geq \varepsilon
$$

by assumption.

For the proof of properties $(i v)$ and $(v)$, let $\theta=\left(\theta_{1}, \ldots, \theta_{\ell}\right) \in\left(\mathbb{S}^{d-1}\right)^{\ell}$. We write $D^{\theta}$ for $D^{\theta_{\ell}} \ldots D^{\theta_{1}}$. Clearly, $f \in C^{r}$ and $\ell \leq r$ implies that $D^{\theta} f \in C^{r-\ell} \subseteq C$. Since $f_{k}$ is at least as smooth as $f$, both $D^{\theta} f$ and $D^{\theta} f_{k}$ are well defined.

We need the well-known fact that $D^{\theta}(f * g)=\left(D^{\theta} f\right) * g$ if $f \in C^{\ell}$ and $g$ has compact support. For $g=g_{1} * \ldots * g_{k}$, we have

$$
\begin{aligned}
\left|D^{\theta} f_{k}(x)-D^{\theta} f_{k}(y)\right| & =\left|\left(\left(D^{\theta} f\right) * g\right)(x)-\left(\left(D^{\theta} f\right) * g\right)(y)\right| \\
& =\mid \int_{\mathbb{R}^{d}}\left[\left(D^{\theta} f(x+t)-D^{\theta} f(y+t)\right] g(t) \mathrm{d} t \mid\right. \\
& \leq \operatorname{Lip}\left(D^{\theta} f\right)\|x-y\|_{2} \int_{\mathbb{R}^{d}} g(t) \mathrm{d} t \\
& =\operatorname{Lip}\left(D^{\theta} f\right)\|x-y\|_{2}
\end{aligned}
$$

for all $x, y \in \mathbb{R}^{d}$. The last equality follows since the $g_{k}$ is normalized. This proves (iv).

For $(v)$, we need to prove that $f_{k} \in C^{r+k}$ with $f_{0}=f \in C^{r}$ by assumption, and then it is enough to show that for all $m \leq r+k$ and all $\theta=\left(\theta_{m}, \ldots, \theta_{1}\right) \in\left(\mathbb{S}^{d-1}\right)^{m}$,

$$
\operatorname{Lip}\left(D^{\theta} f_{k}\right) \leq \frac{1}{\delta \alpha_{k}} \operatorname{Lip}\left(D^{\bar{\theta}} f_{k-1}\right)
$$

where $\bar{\theta}=\left(\theta_{m-1}, \ldots, \theta_{1}\right) \in\left(\mathbb{S}^{d-1}\right)^{m-1}$.

Assume inductively that $f_{k-1} \in C^{m-1}$, which holds for $k=1$. This implies $D^{\bar{\theta}}\left(f_{k-1} * g_{k}\right)=\left(D^{\bar{\theta}} f_{k-1}\right) * g_{k}$, and

$$
\begin{aligned}
D^{\theta} f_{k}(x) & =D^{\theta_{m}}\left(\left(D^{\bar{\theta}} f_{k-1}\right) * g_{k}\right)(x) \\
& =D^{\theta_{m}}\left(\frac{1}{\lambda_{d}\left(B_{k}\right)} \int_{\mathbb{R}^{d}} D^{\bar{\theta}} f_{k-1}(x+t) \mathbb{1}_{B_{k}}(t) \mathrm{d} t\right) \\
& =\frac{1}{\lambda_{d}\left(B_{k}\right)} D^{\theta_{m}}\left(\int_{\theta_{\frac{1}{m}}} \int_{\mathbb{R}} D^{\bar{\theta}} f_{k-1}\left(x+s+h \theta_{m}\right) \mathbb{1}_{B_{k}}\left(s+h \theta_{m}\right) \mathrm{d} h \mathrm{~d} s\right) \\
& =\frac{1}{\lambda_{d}\left(B_{k}\right)} \int_{\theta_{m}^{\frac{1}{m}}} D^{\theta_{m}}\left(\int_{\mathbb{R}} D^{\bar{\theta}} f_{k-1}\left(x+s+h \theta_{m}\right) \mathbb{1}_{B_{k}}\left(s+h \theta_{m}\right) \mathrm{d} h\right) \mathrm{d} s,
\end{aligned}
$$

where $\theta_{m}^{\perp}$ is the hyperplane orthogonal to $\theta_{m}$. For any function $f$ on $\mathbb{R}$ of the form

$$
f(x)=\int_{x-a}^{x+a} g(y) \mathrm{d} y
$$

with some continuous function $g$ we have

$$
f^{\prime}(x)=g(x+a)-g(x-a) .
$$


Therefore, we obtain

$$
\begin{aligned}
D^{\theta} f_{k}(x)=\frac{1}{\lambda_{d}\left(B_{k}\right)} \int_{B_{k} \cap \theta_{m}^{\perp}}[ & D^{\bar{\theta}} f_{k-1}\left(x+s+h_{\max }(s) \theta_{m}\right) \\
& \left.-D^{\bar{\theta}} f_{k-1}\left(x+s-h_{\max }(s) \theta_{m}\right)\right] \mathrm{d} s
\end{aligned}
$$

with

$$
h_{\max }(s)=\max \left\{h \geq 0 \mid s+h \theta_{m} \in B_{k}\right\} .
$$

For each $s \in B_{k} \cap \theta_{m}^{\perp}$, define the points $s_{1}=s+h_{\max }(s) \theta_{m} \in B_{k}$ and $s_{2}=s-h_{\max }(s) \theta_{m} \in B_{k}$. Then

$$
\begin{aligned}
\left|D^{\theta} f_{k}(x)-D^{\theta} f_{k}(y)\right| \leq \frac{1}{\lambda_{d}\left(B_{k}\right)} \int_{B_{k} \cap \theta_{m}^{\perp}}\left[\mid D^{\bar{\theta}} f_{k-1}\left(x+s_{1}\right)-D^{\bar{\theta}} f_{k-1}\left(x+s_{2}\right)\right. \\
\left.-D^{\bar{\theta}} f_{k-1}\left(y+s_{1}\right)+D^{\bar{\theta}} f_{k-1}\left(y+s_{2}\right) \mid\right] \mathrm{d} s \\
\leq \frac{1}{\lambda_{d}\left(B_{k}\right)} \int_{B_{k} \cap \theta_{m}^{\perp}}\left[\left|D^{\bar{\theta}} f_{k-1}\left(x+s_{1}\right)-D^{\bar{\theta}} f_{k-1}\left(y+s_{1}\right)\right|\right. \\
\left.+\left|D^{\bar{\theta}} f_{k-1}\left(x+s_{2}\right)-D^{\bar{\theta}} f_{k-1}\left(y+s_{2}\right)\right|\right] \mathrm{d} s \\
\leq \frac{2 \lambda_{d-1}\left(B_{k} \cap \theta_{m}^{\perp}\right)}{\lambda_{d}\left(B_{k}\right)} \operatorname{Lip}\left(D^{\bar{\theta}} f_{k-1}\right)\|x-y\|_{2} .
\end{aligned}
$$

In particular, this shows the implication

$$
f_{k-1} \in C^{m-1} \Longrightarrow f_{k} \in C^{m}
$$

for all $k \in \mathbb{N}$. Taking $m=r+k$ we have $f_{k} \in C^{r+k}$, as claimed.

For $m \leq r+k$, it remains to bound $2 \lambda_{d-1}\left(B_{k} \cap \theta_{m}^{\perp}\right) / \lambda_{d}\left(B_{k}\right)$. Recall that $B_{k}$ is a ball with radius $\delta \alpha_{k} \sqrt{d}$ and that $V_{d}$ is the volume of the Euclidean unit ball in $\mathbb{R}^{d}$. We obtain from (7) that

$$
\frac{2 \lambda_{d-1}\left(B_{k} \cap \theta_{m}^{\perp}\right)}{\lambda_{d}\left(B_{k}\right)}=\frac{2\left(\delta \alpha_{k} \sqrt{d}\right)^{d-1}}{\left(\delta \alpha_{k} \sqrt{d}\right)^{d}} \frac{V_{d-1}}{V_{d}}=\frac{2}{\delta \alpha_{k} \sqrt{d}} \frac{V_{d-1}}{V_{d}} \leq \frac{1}{\delta \alpha_{k}} .
$$

This concludes the proof that

$$
\operatorname{Lip}\left(D^{\theta_{\ell+j}} D^{\theta_{\ell+j-1}} \ldots D^{\theta_{1}} f_{k}\right) \leq\left(\prod_{i=1}^{j} \frac{1}{\delta \alpha_{k+1-i}}\right) \operatorname{Lip}\left(D^{\theta_{\ell}} D^{\theta_{\ell-1}} \ldots D^{\theta_{1}} f\right)
$$

but since the order of convolution is arbitrary, we obtain in the same way,

$$
\operatorname{Lip}\left(D^{\theta_{\ell+j}} D^{\theta_{\ell+j-1}} \ldots D^{\theta_{1}} f_{k}\right) \leq\left(\prod_{i \in J} \frac{1}{\delta \alpha_{i}}\right) \operatorname{Lip}\left(D^{\theta_{\ell}} D^{\theta_{\ell-1}} \ldots D^{\theta_{1}} f\right),
$$

for all $J \subset\{1, \ldots, k\}$ with $\# J=j$. In particular, this implies $(v)$. 


\section{MAin Results}

Let $\mathcal{P}=\left\{x_{1}, \ldots, x_{n}\right\} \subset \mathbb{R}^{d}$ be a collection of $n$ points. As pointed out in the introduction, we want to construct functions that vanish at $\mathcal{P}$ and have a large integral. For this, we choose

$$
f_{0}(x)=\min \left\{1, \frac{1}{\delta \sqrt{d}} \operatorname{dist}\left(x, \mathcal{P}_{\delta}\right)\right\} \quad \text { for all } \quad x \in \mathbb{R}^{d},
$$

where

$$
\mathcal{P}_{\delta}=\bigcup_{i=1}^{n} B_{\delta}^{d}\left(x_{i}\right)
$$

and $B_{\delta}^{d}\left(x_{i}\right)$ is the ball with center $x_{i}$ and radius $\delta \sqrt{d}$.

The function $\operatorname{dist}\left(\cdot, \mathcal{P}_{\delta}\right)$ is Lipschitz with constant 1 . Hence, for $\delta \leq 1$,

$$
\operatorname{Lip}\left(f_{0}\right)=\frac{1}{\delta \sqrt{d}}
$$

Additionally, $f_{0}(x)=0$ for all $x \in \mathcal{P}_{\delta}$ by definition.

Using these facts we can apply Theorem 1 to prove the curse of dimensionality for the following class of functions that are defined on $\mathbb{R}^{d}$. For a fixed $r \in \mathbb{N}$, we now take $\alpha_{1}=\cdots=\alpha_{r}=\frac{1}{r}$ and define

$$
F_{d, r, \delta}=\left\{f: \mathbb{R}^{d} \rightarrow \mathbb{R} \mid f \in C^{r} \text { satisfies (10)-(12) }\right\},
$$

where

$$
\begin{aligned}
\|f\| & \leq 1, \\
\operatorname{Lip}(f) & \leq \frac{1}{\delta \sqrt{d}}, \\
\forall k \leq r: \max _{\theta_{1}, \ldots, \theta_{k} \in \mathbb{S}^{d-1}} \operatorname{Lip}\left(D^{\theta_{1}} \ldots D^{\theta_{k}} f\right) & \leq \frac{1}{\delta \sqrt{d}}\left(\frac{r}{\delta}\right)^{k} .
\end{aligned}
$$

Theorem 2. For any $r \in \mathbb{N}$ and $\delta \in(0,1]$,

$$
n\left(\varepsilon, F_{d, r, \delta}\right) \geq(1-\varepsilon)\left\{\begin{array}{ll}
1 & \text { for } d=1, \\
(\delta \sqrt{18 e \pi})^{-d} & \text { for } d \geq 2,
\end{array} \quad \text { for all } \varepsilon \in(0,1) .\right.
$$

Hence the curse of dimensionality holds for the class $F_{d, r, \delta}$ for $\delta<1 / \sqrt{18 e \pi}$.

This result shows that the growth rate of $n\left(\varepsilon, F_{d, r, \delta}\right)$ in $d$ can be arbitrarily large if we choose $\delta$ small enough.

Proof. Since the initial error for the classes $F_{d, r, \delta}$ is 1 , we obtain $n\left(\varepsilon, F_{d, r, \delta}\right) \geq 1$ for all $\varepsilon \in(0,1)$. This proves the statement for $d=1$.

For $d \geq 2$, we use Theorem 1 with $k=r, \Omega=\mathcal{P}$ and $f_{r}(x)=f_{0} * g_{1} * \ldots * g_{r}(x)$. Here, the $g_{j}$ 's are as in Theorem 11 Recall that we have chosen $\alpha_{1}=\ldots=\alpha_{r}=1 / r$ and $\alpha_{j}=0$ for $j>r$. The properties of the initial function $f_{0}$ and Theorem 1 immediately imply that $f_{r}$ satisfies (10)-(12). It remains to bound its integral. Note that $f_{0}(x)=1$ for all $x \notin \mathcal{P}_{2 \delta}$. Clearly, $f_{r}(x) \geq 0$ for all $x \in \mathbb{R}^{d}$. Since $f_{r}(x)$ depends only on the values $f_{0}(x+t)$ for $t \in \mathbb{R}^{d}$ with $\|t\|_{2} \leq \delta \sqrt{d}$, it follows that 
$f_{r}(x)=1$ for $x \notin \mathcal{P}_{3 \delta}$. We thus obtain

$$
\begin{aligned}
\int_{D_{d}} f_{r}(x) \mathrm{d} x & \geq \int_{D_{d} \backslash \mathcal{P}_{3 \delta}} f_{r}(x) \mathrm{d} x=1-\lambda_{d}\left(\mathcal{P}_{3 \delta} \cap D_{d}\right) \\
& \geq 1-\lambda_{d}\left(\mathcal{P}_{3 \delta}\right) \geq 1-n \lambda_{d}\left(B_{3 \delta}^{d}\right) \\
& >1-\frac{n(3 \delta \sqrt{2 e \pi})^{d}}{\sqrt{\pi d}} \\
& >1-n(3 \delta \sqrt{2 e \pi})^{d}
\end{aligned}
$$

where the next to last inequality follows from the bound in (6). Hence $\int_{D_{d}} f_{r}(x) \mathrm{d} x$ $\leq \varepsilon$ implies that

$$
n \geq(1-\varepsilon)(\delta \sqrt{18 \varepsilon \pi})^{-d} .
$$

Since this holds for arbitrary $\mathcal{P}$, the result follows.

By Theorem 2, we know how the parameter $\delta$ comes into play. For $p>0$, let

$$
\delta=\frac{1}{\sqrt{18 e \pi}} d^{-p /(r+1)} .
$$

For this $\delta$, we obtain a somehow stronger form of the curse of dimensionality for the class

$$
\widetilde{F}_{d, r, p}=\left\{f: \mathbb{R}^{d} \rightarrow \mathbb{R} \mid f \in C^{r} \text { satisfies (13)-(15) }\right\},
$$

where

(15) $\forall k \leq r: \max _{\theta_{1}, \ldots, \theta_{k} \in \mathbb{S}^{d-1}} \operatorname{Lip}\left(D^{\theta_{1}} \ldots D^{\theta_{k}} f\right) \leq d^{-\frac{1}{2}+\frac{p(k+1)}{r+1}} r^{k}(\sqrt{18 e \pi})^{k+1}$.

Theorem 3. For any $r \in \mathbb{N}$ and $p>0$,

$$
n\left(\varepsilon, \widetilde{F}_{d, r, p}\right) \geq(1-\varepsilon) d^{p d /(r+1)} \quad \text { for all } d \in \mathbb{N} \text { and } \varepsilon \in(0,1) .
$$

Hence the curse of dimensionality holds for the class $\widetilde{F}_{d, r, p}$.

Note that the classes $\widetilde{F}_{d, r, p}$ are contained in the classes

$$
\mathcal{C}_{d}^{r}=\left\{f \in C^{r} \mid\left\|D^{\beta} f\right\| \leq 1 \text { for all }|\beta| \leq r\right\},
$$

if $p<1 / 2$ and $d$ is large enough. This holds if

$$
d \geq\left(r^{r}(18 \mathrm{e} \pi)^{(r+1) / 2}\right)^{1 /(1 / 2-p)} .
$$

From this we easily obtain the main result already stated in the introduction.

Main Theorem. For any $r \in \mathbb{N}$, there exists a constant $c_{r} \in(0,1]$ such that

$$
n\left(\varepsilon, \mathcal{C}_{d}^{r}\right) \geq c_{r}(1-\varepsilon) d^{d /(2 r+3)} \quad \text { for all } d \in \mathbb{N} \text { and } \varepsilon \in(0,1) .
$$

Hence the curse of dimensionality holds for the class $\mathcal{C}_{d}^{r}$. 
Proof. The case $d=1$ is trivial since the initial error for the classes $\mathcal{C}_{d}^{r}$ is again 1 .

For $d \geq 2$, we know from Theorem 3 and the discussion thereafter that $n\left(\varepsilon, \mathcal{C}_{d}^{r}\right) \geq$ $(1-\varepsilon) d^{p d /(r+1)}$ for all $p<1 / 2$ if $d \geq d_{0}$, where $d_{0}=d_{0}(r, p)$ is the right-hand side of (16). This implies

$$
n\left(\varepsilon, \mathcal{C}_{d}^{r}\right) \geq \widetilde{c}_{r, p}(1-\varepsilon) d^{p d /(r+1)} \quad \text { for all } \quad d \geq 2,
$$

with

$$
\widetilde{c}_{r, p}=d_{0}^{-p d_{0} /(r+1)},
$$

which depends only on $r$ and $p$. The choice $p^{*}=(r+1) /(2 r+3)$ yields the result with $c_{r}=\widetilde{c}_{r, p^{*}}$.

Note that $c_{r}$ in the last theorem is super-exponentially small in $r$.

Remark 1. The reader might find it more natural to define classes of functions $F_{d, r}\left(D_{d}\right)$ that are defined only on $D_{d} \subset \mathbb{R}^{d}$. Not all such functions can be extended to smooth functions on $\mathbb{R}^{d}$, and even if they can be extended then the norm of the extended function could be much larger. Our lower bound results for functions defined on $\mathbb{R}^{d}$ can be also applied for functions defined on $D_{d} \subset \mathbb{R}^{d}$ and this makes them even stronger.

Remark 2. Note that the possibility of super-exponential lower bounds on the complexity depends on the definition of the Lipschitz constant. For the class

$$
F_{d}=\left\{f:[0,1]^{d} \rightarrow \mathbb{R} \mid \sup _{x, y \in[0,1]^{d}} \frac{|f(x)-f(y)|}{\|x-y\|_{\infty}} \leq 1\right\},
$$

Sukharev 8 , proved that the product mid-point rule is optimal with error $e_{n}=$ $\frac{d}{2 d+2} n^{-1 / d}$ for $n=m^{d}$. Hence, roughly, $n\left(\varepsilon, F_{d}\right) \approx 2^{-d} \varepsilon^{-d}$ and the complexity is "only" exponential in $d$ for $\varepsilon<1 / 2$.

Remark 3. We mention two results for the very small class

$$
F_{d}=C_{d}^{\infty}=\left\{f \in C^{\infty}\left([0,1]^{d}\right) \mid\left\|D^{\beta} f\right\| \leq 1 \quad \text { for all } \beta \in \mathbb{N}_{0}^{d}\right\} .
$$

O. Wojtaszczyk 10] proved that $\lim _{d \rightarrow \infty} n\left(\varepsilon, F_{d}\right)=\infty$ for every $\varepsilon<1$, hence the problem is not strongly polynomially tractable. It is still open whether the curse of dimensionality holds for this class $F_{d}$. The same class $F_{d}$ was studied for the approximation problem in [6]. For this problem the curse of dimensionality is present even if we allow algorithms that use arbitrary linear functionals.

\section{ACKNOWLEDGMENTS}

We thank Jan Vybíral and Shun Zhang for their valuable remarks.

\section{REFERENCES}

[1] M. Abramowitz and I. A. Stegun, Handbook of Mathematical Functions, National Bureau of Standards Applied Mathematics Series, 55, U.S. Government Printing Office, Washington, DC, tenth printing, 1972. MR0167642 (29 \#4914)

[2] N. S. Bahvalov, Approximate computation of multiple integrals (Russian), Vestnik Moskov. Univ. Ser. Mat. Meh. Astr. Fiz. Him. 1959 (1959), no. 4, 3-18. MR0115275 (22 \#6077)

[3] A. Hinrichs, E. Novak, and H. Woźniakowski, The curse of dimensionality for the class of monotone functions and for the class of convex functions, J. Approx. Theory 163 (2011), no. 8, 955-965, DOI 10.1016/j.jat.2011.02.009. MR2832759 (2012g:41040)

[4] E. Novak, Deterministic and Stochastic Error Bounds in Numerical Analysis, Lecture Notes in Mathematics, vol. 1349, Springer-Verlag, Berlin, 1988. MR971255 (90a:65004) 
[5] E. Novak and H. Woźniakowski, Tractability of Multivariate Problems. Vol. 1: Linear Information, EMS Tracts in Mathematics, vol. 6, European Mathematical Society (EMS), Zürich, 2008. MR2455266(2009m:46037)

[6] E. Novak and H. Woźniakowski, Approximation of infinitely differentiable multivariate functions is intractable, J. Complexity 25 (2009), no. 4, 398-404, DOI 10.1016/j.jco.2008.11.002. MR2542039(2010h:41046)

[7] E. Novak and H. Woźniakowski, Tractability of Multivariate Problems. Volume II: Standard Information for Functionals, EMS Tracts in Mathematics, vol. 12, European Mathematical Society (EMS), Zürich, 2010. MR2676032(2011h:46093)

[8] A. G. Sukharev, Optimal numerical integration formulas for some classes of functions of several variables (English translation), Soviet Math. Dokl. 20 (1979), no. 2, 472-475, 1979. MR $0533623(80 \mathrm{j}: 65016)$

[9] J. F. Traub, G. W. Wasilkowski, and H. Woźniakowski, Information-based Complexity, Computer Science and Scientific Computing, Academic Press Inc., Boston, MA, 1988. With contributions by A. G. Werschulz and T. Boult. MR.958691 (90f:68085)

[10] J. O. Wojtaszczyk, Multivariate integration in $C^{\infty}\left([0,1]^{d}\right)$ is not strongly tractable, J. Complexity 19 (2003), no. 5, 638-643, DOI 10.1016/S0885-064X(03)00069-4. MR2003236 (2004g:65191)

Institut für Mathematik, Universität Rostock, Ulmenstrasse 69, 18051 Rostock, GERMANY

E-mail address: aicke.hinrichs@uni-rostock.de

E-mail address: a.hinrichs@uni-jena.de

Mathematisches Institut, Universität Jena, Ernst-Abbe-Platz 2, 07743 Jena, GerMANY

E-mail address: erich.novak@uni-jena.de

Dipartimento di Matematica, Università Roma Tre, Largo San Leonardo Murialdo 1, 00146 Roma, ITALY

E-mail address: ullrich.mario@gmail.com

Department of Computer Science, Columbia University, New York, New York 10027 - And - Institute of Applied Mathematics, University of Warsaw, ul. Banacha 2, 02-097 WarszaWa, Poland

E-mail address: henryk@cs.columbia.edu 